Abstract T3 Table 1 The effects of ECVE exposure on MMP-9 and CXCL8 release from blood neutrophils after $6 \mathrm{hr}$, and CXCL8 release from alveolar macrophages after $24 \mathrm{hr}$. Data presented as mean (sd)

\begin{tabular}{llllll}
\hline Neutrophils & & \multicolumn{3}{l}{ Macrophages } \\
\hline MMP-9 & & CXCL8 & \multicolumn{3}{c}{ CXCL8 } \\
\hline 0 ECVE & 0.003 ECVE & 0 ECVE & 0.003 ECVE & 0 ECVE & 0.003 ECVE \\
& & 175 & 348 & 1647 & 6000 \\
$44782(25809)$ & $184585(104617)$ & $(248)$ & $(364)$ & $(2457)$ & $(7602)$ \\
\hline
\end{tabular}

We have investigated the effects of e-cigs on human innate immune cells in vitro.

Methods Blood neutrophils from six healthy non-smokers were exposed to e-cig vapour extract (ECVE) for $6 \mathrm{hr}$. MMP-9 and CXCL8 release were measured by ELISA and MMP-9 activity was measured by zymography. p38 MAPK activation was also measured, along with neutrophil shape change and CD11b and CD66b expression by flow cytometry. Finally, we measured CXCL8 release from alveolar macrophages isolated from resected lung tissue from three ex-tobacco smokers exposed to ECVE for $24 \mathrm{hr}$.

Results Exposure of neutrophils to ECVE increased MMP-9 and CXCL8 release with the maximal effect observed at an optical density (OD) of 0.003 (Table 1). This was observed along with an increase in MMP-9 gelatinase activity and increased p38 MAPK activation.

Furthermore, neutrophil shape change, and dual CD11b and CD66b expression increased in response to ECVE treatment compared to untreated cells.

Following a similar trend, 0.003 (OD) ECVE caused an increase in CXCL8 release from alveolar macrophages.

Discussion We have shown that e-cig exposure causes an inflammatory response from neutrophils and macrophages. The effects discussed here are similar to those caused by tobacco cigarettes. Based on these findings, the use of e-cigs may pose a risk to public health.

\section{T4 PNEUMOCOCCAL CONJUGATE VACCINE REDUCES RATE, DENSITY AND DURATION OF EXPERIMENTAL HUMAN PNEUMOCOCCAL COLONISATION: FIRST HUMAN CHALLENGE TESTING OF A PNEUMOCOCCAL VACCINE}

${ }^{1} \mathrm{AM}$ Collins, ${ }^{2} \mathrm{AD}$ Wright, ${ }^{3} \mathrm{E}$ Mitsi, ${ }^{3} \mathrm{JF}$ Gritzfeld, ${ }^{1} \mathrm{C}$ Hancock, ${ }^{1} \mathrm{D}$ Shaw, ${ }^{3} \mathrm{SH}$ Pennington, ${ }^{3} \mathrm{~B}$ Morton, ${ }^{3} \mathrm{DM}$ Ferreira, ${ }^{3} \mathrm{SB}$ Gordon. ${ }^{1}$ Respiratory Infection Group, Royal Liverpool and Broadgreen University Hospital Trust, Liverpool, UK; ${ }^{2}$ Respiratory Infection Group, Royal Liverpool and Broadgreen University Hospital Trust and Local Comprehensive Research Network, Liverpool, UK; ${ }^{3}$ Respiratory Infection Group, Liverpool School of Tropical Medicine, Pembroke Place, L3 5QA, Liverpool, UK

\subsection{6/thoraxjnl-2014-206260.4}

Objective To determine the effect of the Pneumococcal Conjugate Vaccine (PCV-13) on experimental pneumococcal colonisation compared to Hepatitis A vaccination (control) in healthy participants.

Design Double blind randomised controlled trial.

Setting Clinical Research Unit in the Royal Liverpool University Hospital.

Participants 99 healthy participants aged 18-50 years were randomly assigned to receive PCV-13 $(\mathrm{n}=49)$ or Hepatitis A $(\mathrm{n}=$ 50) vaccination according to a randomisation plan in blocks of ten.

Interventions Participants previously vaccinated with PCV or control were inoculated after 4 weeks with $80,000 \mathrm{C} \mathrm{FU/100} \mu \mathrm{l}$

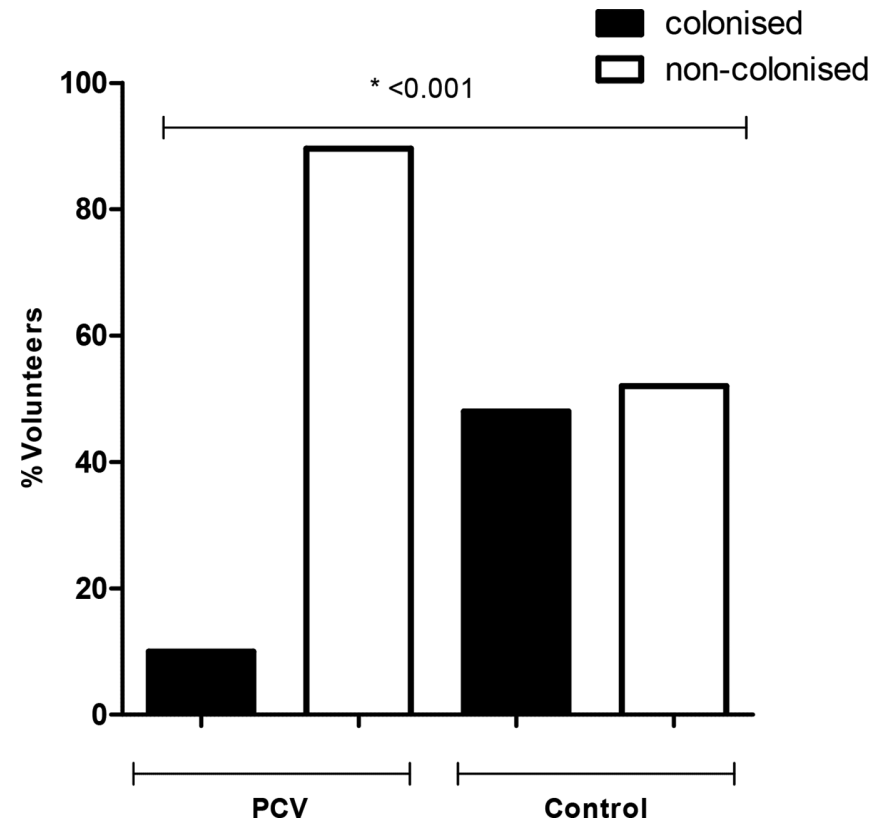

Abstract T4 Figure 1 Percentage of participants' colonised and noncolonised by $6 \mathrm{~B}$ pneumococcus assessed using classical microbiology according to vaccination group at any time point

pneumococcal bacteria (6B) $100 \mu \mathrm{l}$ per naris. Participants were followed up for 21 days to determine pneumococcal colonisation by culture of nasal wash samples.

Main outcome measures The primary outcome measure was the culture of type $6 \mathrm{~B}$ pneumococcus at either day 2, 7, 14 or 21 following inoculation. Secondary outcome measures included the density and duration of pneumococcal colonisation post inoculation of $6 \mathrm{~B}$ and the presence of any other naturally acquired pneumococcal strains.

Results The PCV group showed a significantly reduced experimental colonisation rate 5/48 compared to the control group 23/ $48(<0.001)$ [Figure 1]. Both the density and duration of colonisation were reduced in the PCV group compared to the control group following inoculation. The area under the density-time curve (total exposure) was significantly reduced in the PCV compared to control group (mean 8902 vs $267580 \mathrm{p}=0.0179$ ).

Conclusion PCV reduces pneumococcal colonisation rate, density and duration in healthy adults. The Experimental Human Pneumococcal Colonisation (EHPC) model is a safe, effective and efficient method of analysing the efficacy of vaccination on pneumococcal colonisation. We suggest that this novel EHPC model can now be used as a platform for future pneumococcal vaccine testing, using small sample sizes and shorter time scales than community studies in order to reduce time and cost to market. We recommend that carriage rate, density and duration are all measured in these studies.

Trial registration.

EudraCT: 2012-005141-20.

ISRCTN: 45340436.

\section{T5 OPG REGULATES PULMONARY ARTERIAL SMOOTH MUSCLE CELL PROLIFERATION AND THE EXPRESSION OF PAH-ASSOCIATED GENES VIA FAS}

S Dawson, J Pickworth, AMK Rothman, J Iremonger, N Arnold, S Francis, A Lawrie. University of Sheffield, Sheffield, UK

10.1136/thoraxjnl-2014-206260.5 